\title{
Clinical trials in psychiatry: Obtaining the evidence
}

\author{
Sue Hawkridge is a senior specialist psychiatrist in the Department of Psychiatry at \\ the University of Stellenbosch. Her areas of interest include early-onset psychotic \\ disorders and paediatric psychopharmacology. \\ Robin Emsley is Head of the Department of Psychiatry, University of Stellenbosch. \\ His areas of research are psychopharmacology and schizophrenia.
}

Clinical trials have become a commonplace in medical practice in South Africa. They are seen as a method of obtaining otherwise unavailable new medications for state patients in a cash-strapped health care system, as a way of supplementing the incomes and thus retaining the services of highly marketable specialist staff in state hospitals, as access to academic medicine for practitioners isolated from teaching institutions, and as additional income for private practitioners who need to subsidise patients on inadequate medical aids. Perhaps some also see them as a way of enriching already well-paid practitioners, although those who have participated in them might agree that a short overseas locum is more profitable and a good deal less trouble.

There are, however, sound scientific reasons for the inclusion of clinical trials in medical practice, particularly in psychiatry.

\section{Why are clinical trials necessary?}

Clinical trials are needed in order to establish the efficacy and safety of any treatment. There is simply no place in clinical medical practice for compounds which have not been proved effective and safe. New drugs are being discovered and developed at a greater rate than at any time in the history of medicine. At the same time, quackery and faddism continue to flourish. Therapeutic claims with no scientific backing are made for many substances, and these substances are marketed to a largely uninformed and gullible public (remember Virodene?). While regulatory authorities make valiant efforts to register and control the sale of any substance claimed to have an effect on any system of the body, 'nutritional supplements', 'natural remedies', 'herbal treatments' and 'traditional medicines' can disguise a plethora of potentially harmful or inactive substances. In a jungle of false claims and exaggerated testimonies, it is imperative that clinical medicine retain its

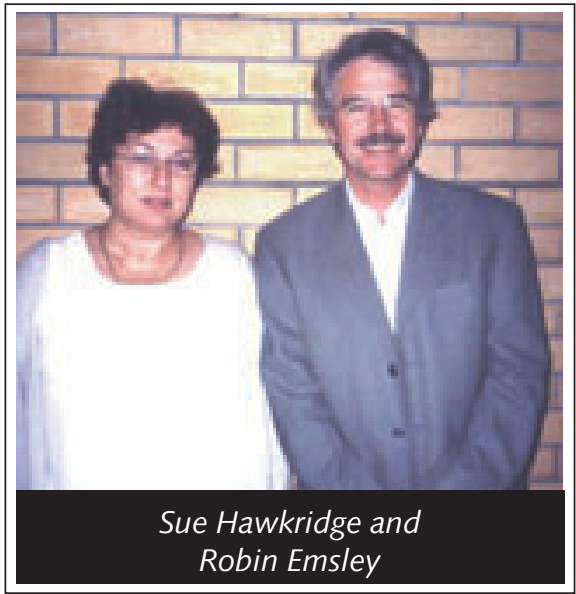

credibility, and the only method currently accepted by all major disciplines is the clinical trial.

\section{The search for new drugs}

Drug discoveries have frequently been accidental. In the field of psychopharmacology the process has been particularly haphazard and fortuitous. Sometimes a slight structural change in a known compound gives rise to unlooked for but important major functional alterations. Chlorpromazine, for instance, was an unexpected outcome of the search for a better antihistamine. Iproniazide is an analogue of isoniazide, an early anti-tuberculosis drug. The first tricyclic antidepressant was discovered when the sulphur in chlorpromazine was substituted by a $\mathrm{C}=\mathrm{C}$ molecule. However, the past two decades or so have seen a dramatic change in the development of new psychotropic agents. Thanks to technological advances, so-called 'designer drugs' lof which Prozac was the prototype) are now being produced in laborato- 
ries around the world. Molecules are being manipulated and moulded to create compounds with increasingly selective effects on central nervous system neurotransmitters in the hope of developing more effective treatments with fewer untoward side-effects. The search for new and more effective psychotropic drugs has also been accelerated in the last few decades by the recognition that psychiatric disorders are very prevalent, and that a much broader spectrum of these disorders than previously recognised can benefit from medication.

\section{Drug development}

A drug development programme is an extremely complex process, comprising many phases over a number of years. In the preclinical phase, researchers attempt to create molecules which, according to current aetiological models, would stand a good chance of reversing causal factors or ameliorating symptoms. These molecules are then tested in animal studies, and receptor binding profiles are defined. These processes assist in predicting likely clinical applications and the anticipated sideeffect profile. An example in psychiatry would be the development of the new-generation antipsychotic drugs. The recognition of certain efficacy and side-effect advantages of clozapine over the conventional antipsychotics led to pharmaceutical laboratories delineating its receptor binding profile and identifying the aspects that are most likely to be responsible for these advantages. The result was the development of several new agents with relatively modest dopamine $D_{2}$-receptor blocking activity combined with potent $5 \mathrm{HT}_{2}$ blockade. ${ }^{2}$ These agents have now become first-choice treatments for psychosis in much of the developed world.

There are four clinical phases of drug development. In phase I, undertaken in healthy human volunteers, the safety and appropriate dosage of the new drug are established. In phase II, patients with the relevant disorder are recruited and efficacy of the new drug compared with placebo is assessed. Further information on adverse events and effective dosage is also collected. In phase III studies, large-scale multi-centre trials are carried out, and efficacy and safety relative to established treatments and/or placebo are assessed. Most multinational studies conducted in South Africa fall within this category. Registration of the drug follows successful phase III studies. Phase IV studies are carried out after registration, in order to assess ongoing efficacy and potential long-term sideeffects, as well as possible applications of the agent in specific patient populations, such as those with refractory illness.

\section{Trial design}

Various types of trial design may be used, including case reports, open-label trials, naturalistic studies and controlled studies. However, controlled studies provide the best evidence of safety and efficacy. Controlled studies are randomised and blinded (single- or double-blind), and compare the test compound to placebo, an active comparator, or both. Dose can be fixed or flexible over a certain range, and duration may be acute (usually 6 - 12 weeks) or long term (usually 6 - 12 months, or longer).

There are several critical issues in designing a clinical trial, and the following fundamental questions have to be answered. It must be established what the likely benefits from the drug might be Against this the possible risks must be identified. The correct lboth safe and effective) dose range has to be defined. Evidence must be obtained of how the new drug compares with alternative treatments. Lastly, it is important to identify whether or not there are specific subgroups of patients most likely to benefit from the new drug

$11 e_{\text {double-blind, clinical trial was one }}^{\text {introduction of the randomised, }}$ of the major advances in the development of medical science.' J M Kane ${ }^{3}$

In modern medical research, the randomised controlled trial (RCT) has become the gold standard for the assessment of any new treatment. While open-label, naturalistic studies provide certain information, demonstration of efficacy can only be confidently accomplished by means of the RCT. The inclusion of a placebo group in the RCT is regarded by regulatory authorities as critical in the establishment of assay sensitivity. In other words, demonstration of non-inferiority to established treatment does not necessarily imply efficacy, as the placebo effect of medications may be substantial and misleading. In fact, approximately one in three trials of registered antidepressants failed to differentiate the active drug from placebo! ${ }^{4}$

\section{The placebo effect}

For centuries, placebos have been regarded as powerful deceptive therapies. However, from the middle of the 20th century, placebos have been used to distinguish the specific from the nonspecific ingredients in treatments. The placebo response has more 
recently been the subject of study in its own right. Placebo is known to improve subjective and objective measures of disease in $30-40 \%$ of a wide range of conditions, e.g. allergies/asthma, alopecia, Parkinson's disease, erectile dysfunction, osteoporosis and weight loss. According to a recent meta-analysis of RCTs of antitussive agents, $85 \%$ of the reduction in cough is related to treatment with placebo, and only 15\% attributable to the active ingredient. ${ }^{5}$ The placebo effect is poorly understood, and until recently, under-researched. Possible mechanisms have been postulated, including expectation and conditioning. Frontal dopaminergic reward systems may be important. The expectation of reward (i.e. clinical benefit) seems to be particularly relevant. The brain has processes, functional salutogenic mechanisms, that contribute to health by enhancing one's outlook on life to the benefit of one's health. Beliefs need not even be rational or realistic, as shown in studies of phenomena such as faith healing and the placebo effect. ${ }^{6}$ Until recently, little was known about the neurological pathways of these functional salutogenic mechanisms. A thought-provoking study explored the functional anatomy of the placebo effect by studying regional brain glucose metabolism by means of positron emission tomography. The study compared patients with major depressive disorder who had responded to fluoxetine treatment with those who had responded to placebo treatment. The placebo-responders demonstrated frontal cortical activation and limbic-paralimbic inhibition in specific regions. Fluoxetine responders demonstrated remarkably similar changes in these regions, although of greater magnitude. There were also changes that were specific to fluoxetine responders, namely subcortical inhibition, and interestingly, early brainstem activation. An early change that differentiated placebo responders from placebo non-responders was posterior cingulate activation after 1 week of treatment. The authors propose a model of 'bottom-up' cascade effects of antidepressants and 'top-down' (i.e. from cortex) in placebo responders.?

The importance of including a placebo arm in antidepressant trials was clearly demonstrated in a multi-centre, non-pharmaceutical industry sponsored RCT investigating the efficacy of Hypericum perforatum (St John's wort). The study, conducted in a large sample ( $N=338$ ) over 8 weeks, included a placebo arm as well as an established antidepressant, sertraline, as comparator. Considerable reduction in depression scores was recorded in the hypericum group, and if this had been an uncontrolled study, the investigators might well have concluded that hypericum was an effective antidepressant. But the placebo group displayed a similar reduction in depressive symptomatology - i.e. hypericum was no better than placebo. However, the sertraline group also did not differ significantly from either placebo or hypericum. This, then, was a 'failed study'. ${ }^{8}$

\section{Reasons for retention of the placebo arm}

Retention of a placebo arm in RCTs has been the subject of much ethical deliberation. Those arguing against its use cite the World Medical Association Helsinki Declaration (2000) stating that placebos can never be justified where an effective treatment exists. Those arguing for its retention state that placebo is acceptable as long as it is not associated with a significant risk of additional permanent morbidity. In fact, it has been stated that not to include placebo is more unethical because of the risk of approving ineffective medications. The use of a placebo arm in controlled clinical trials eliminates the risk to public health entailed in the approval of ineffective medications. There is no evidence that treatment delay or assignment to a placebo arm results in permanent harm to psychiatric patients. The risk of suicide due to withholding active antidepressant treatment has been a major concern. However, in an analysis of Food and Drug Administration summary reports of 48277 subjects participating in antidepressant RCTs, based on patient exposure years, suicide rates were similar among subjects assigned to antidepressants and placebo. ${ }^{9}$

In recent years, the placebo response in antidepressant trials has increased considerably, and is substantial in most studies. ${ }^{10}$ In fact, a placebo response of $>50 \%$ is now commonplace in antidepressant RCTs. This has made it much more difficult to differentiate between an active compound and placebo. Much attention is now given to conducting studies in a manner that is most likely to discriminate between placebo and active medication. This includes appropriate selection of subjects, i.e. excluding likely placebo-responders and patients with refractory conditions, maintaining an attitude that will not unduly raise the expectation of improvement, and rigorous and consistent application of rating scale rules. Early withdrawals due to investigator anxiety are avoided as far as possible, as are assumptions about the treatment being tested. In order to provide an adequate frame of reference, sufficient numbers of patients per investigator are included.

\section{Conclusion}

There are some aspects of clinical trials as currently conducted that raise legitimate concerns among researchers. The policy of accelerating drug development programmes in order to extend the period of patent protection should be revisited. The resultant 
pressure to recruit subjects as rapidly as possible is probably a major factor in the increased placebo response rates, as unsuitable subjects are more likely to be included. However, provided that individual clinicians make every effort to conduct trials both meticulously and ethically, clinical trials enhance the quality of care available to patients who might otherwise not have access to newer therapies. Based on currently available information, the RCT remains the best method of proving the efficacy and safety of newly developed drugs, and a placebo arm is a necessary evil until the advent of a better alternative. In the meantime, it is incumbent upon researchers to attempt to improve the RCT design rather than reject it.

\section{Robin Emsley}

\section{Sue Hawkridge}

\section{Department of Psychiatry}

University of Stellenbosch

Tygerberg

W Cape
1. Yuwiler A, Wetterberg L. Guide to Psychiatric Research. Washington, DC: CRC Press, 2000.

2. Meltzer HY, Li Z, Kaneda Y, Ichikawa J. Serotonin receptors : their key role in drugs to treat schizophrenia. Prog Neuropsychopharmacol Biol Psychiatry 2003; 27: 11 59-1 172.

3. Kane J. Progress defined - short-term efficacy, long-term effectiveness. Int Clin Psychopharmacol 2001; 16: Suppl 1; S1-S8

4. Laughren TP. The scientific and ethical basis for placebo-controlled trials in depression and schizophrenia: an FDA perspective. Eur Psychiatry 2001; 16: $418-423$.

5. Eccles R. The powerful placebo in cough studies? Pulm Pharmacol Ther 2002; 15: 251 1-252.

6. Stewart-Williams S. The placebo puzzle: putting together the pieces. Health Psychol 2004 23: $198-206$.

7. Mayberg HS, Silva JA, Brannan SK, Tekell JL, et al. The functional neuroanatomy of the place bo effect. Am J Psychiatry 2002; 159: 728-737.

8. Hypericum Depression Trial Study Group. Effect of Hypericum perforatum (St John's Wort) in major depressive disorder. JAMA 2002; 287: 1807-1814.

9. Khan A, Khan S, Kolts R, Brown WA. Suicide rates in clinical trials of SSRIs, other antidepressants, and placebo: analysis of FDA reports. Am J Psychiatry 2003; 160: 790-792.

10. Walsh BT, Seidman SN, Sysko R, Gould M. Placebo response in studies of major depression: variable, substantial, and growing. JAMA 2002; 287: 1840-1847. 